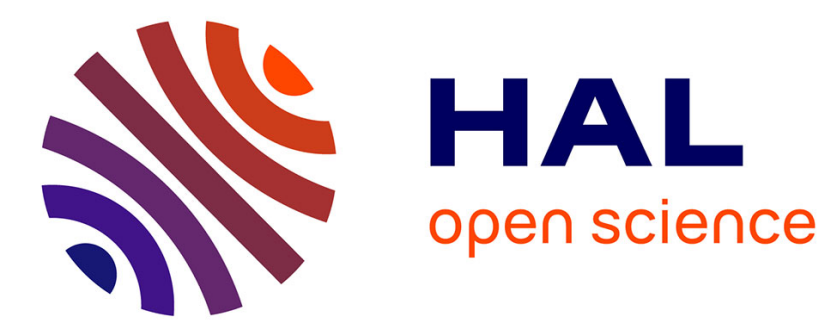

\title{
Direct Observation of Shear-Induced Structures in Wormlike Micellar Solutions by Freeze-Fracture Electron Microscopy
}

\author{
S L Keller, Philippe Boltenhagen, D J Pine, J A Zasadzinski
}

\section{To cite this version:}

S L Keller, Philippe Boltenhagen, D J Pine, J A Zasadzinski. Direct Observation of Shear-Induced Structures in Wormlike Micellar Solutions by Freeze-Fracture Electron Microscopy. Physical Review Letters, 1998. hal-02407740

\author{
HAL Id: hal-02407740 \\ https://hal.science/hal-02407740
}

Submitted on 12 Dec 2019

HAL is a multi-disciplinary open access archive for the deposit and dissemination of scientific research documents, whether they are published or not. The documents may come from teaching and research institutions in France or abroad, or from public or private research centers.
L'archive ouverte pluridisciplinaire HAL, est destinée au dépôt et à la diffusion de documents scientifiques de niveau recherche, publiés ou non, émanant des établissements d'enseignement et de recherche français ou étrangers, des laboratoires publics ou privés. 


\title{
Direct Observation of Shear-Induced Structures in Wormlike Micellar Solutions by Freeze-Fracture Electron Microscopy
}

\author{
S. L. Keller, ${ }^{1}$ P. Boltenhagen, ${ }^{2}$ D. J. Pine, ${ }^{1}$ and J. A. Zasadzinski ${ }^{1}$ \\ ${ }^{1}$ Departments of Chemical Engineering and Materials Science, University of California, Santa Barbara, California $93106-5080$ \\ ${ }^{2}$ L.U.D.F.C. URA no. 851 CNRS, 4 rue Blaise Pascal, 67070 Strasbourg Cedex, France
}

(Received 2 July 1997)

\begin{abstract}
We report the first direct observation of shear-induced structures in low concentration wormlike micellar solutions. The structures consist of micron-sized patches which are much larger than individual equilibrium micelles and occupy 5\%-10\% of the fracture area. Such morphologies are consistent with recent reports of increased light scattering under shear flow. The structures have a stippled or spongelike texture and contain more surfactant than the background micellar solution. These patches form aggregates spanning hundreds of microns and are consistent with the shear thickening observed in these systems. [S0031-9007(98)05452-0]
\end{abstract}

PACS numbers: 83.50.Qm, 61.16.Bg, 83.50.By, 83.70.Hq

Wormlike micelles or living polymers are one of an array of dynamic equilibrium structures formed by surfactant self-assembly. The surfactants aggregate in $5 \mathrm{~nm}$ diameter cylinders which can grow microns long [1]. Although they resemble polymers, wormlike micelles have a more dynamic structure which breaks and re-forms reversibly [2]. The rheological behavior of dilute solutions of very long $(>1 \mu \mathrm{m})$ wormlike micelles is complex and fascinating. When sheared below a critical shear rate which depends on temperature and on surfactant and salt concentrations, dilute wormlike micellar solutions shear thin. The viscosity or shear stress decreases with shear rate, as in typical polymer solutions [3-5]. In contrast, above a critical shear rate, micellar solutions exhibit time-dependent behavior. Initially the solutions thin, and after an induction period shear thickens. During the induction period, light scattering and flow birefringence show that the wormlike micelles are weakly aligned with the stress $[3,4]$. The induction period can last many minutes $\left(\sim 10^{4}\right.$ strain units $)$ and decreases with increasing shear rate. This behavior is poorly understood $[4,6]$.

After the induction period, both the viscosity and shear stress rise sharply. This shear thickening is correlated with the growth of features that scatter light strongly [6]. Simultaneously, flow alignment is observed by small-angle light scattering [3], neutron scattering [7], and flow birefringence $[4,5,8,9]$. Eventually, the viscosity, stress, light scattering, and birefringence plateau. At this point the highly scattering regions span the entire shear cell and individual structures rip apart and grow again. Associated with these dynamic structures are fluctuations in the stress and viscosity about the plateau value $[3-6,9,10]$. Upon the cessation of shear, the stress, viscosity, and birefringence relax slowly ( $\sim 100 \mathrm{sec}$ in our samples) and the sample recoils elastically $[3,11]$. The slow relaxation time and high recoverable stress (up to 5000\%) are surprising because these systems contain $<0.1 \%$ surfactant [3]. Even more surprising, sheared micellar solutions take very long, sometimes hours, to return to an equilibrium unsheared state [4].
The microstructures responsible for these phenomena have been broadly named shear-induced structures or phases (SIS or SIP). Theories have suggested that shear thickening occurs because free micelles join a transient network [12] or because micelles elongate $[13,14]$. The SIP has been variously described as a highly entangled (or branched) [6] three-dimensional network [9] of a viscous gel phase $[3,10]$ which is strongly aligned and consists of structures much larger than individual micelles $[8,15]$. Many models have been proposed, including that the microscopic structures are stringlike [15], highly aligned rods [14], small clusters of micelles [9], pearl strings of micelles, pseudonematic domains [8], or layers of entangled wormlike micelles at high and low shear rates following a reptation-reaction model [16]. The lack of direct, experimental evidence to discriminate between various models has greatly impeded progress in understanding these phenomena.

We use freeze-fracture electron microscopy (FFEM) to observe unsheared and sheared wormlike micelles at low concentrations. FFEM resolves length scales from nanometers (micelles) to microns (patches of new structures). Freeze-fracture's advantage over scattering methods is that it provides direct real-space information about ordered and disordered regions simultaneously [17]. Our principle result is that shear causes the formation of new micron-sized structures that are much larger than individual micelles and consistent with light scattering results $[3,6,10]$. Patches of new structure connect in larger superstructures spanning hundreds of microns. These percolating networks are likely responsible for an increase in viscosity [3-6,8-10]. The new structures are richer in surfactant than the surrounding micellar solution and have a submicron stippled or spongelike texture. Aligned and unaligned wormlike micelles coexist with the new superstructures.

We used two cationic surfactants, tris (2-hydroxyethyl) tallowalkyl ammonium acetate (TTAA) and cetyltrimethyl ammonium bromide (CTAB) [18]. Adding aromatic 
counterions (sodium salicylate, NaSal) creates very long micelles $(>1 \mu \mathrm{m})[1,19]$. Dilute solutions of the micelles form shear-induced phases and shear thicken. We conducted FFEM on both unsheared and sheared equimolar solutions of $10 \mathrm{mM}$ TTAA/NaSal and of 0.55 , 1.37, $13.7 \mathrm{mM} \mathrm{CTAB} / \mathrm{NaSal}$. For unsheared samples, $1 \pm 0.5 \mu 1$ were deposited between $\mathrm{Cu}$ planchettes and the resulting $200-\mu \mathrm{m}$-thick sandwich equilibrated $10 \mathrm{~min}$ before it was vitrified.

Samples were sheared in a cone-plate rheometer (Brookfield) at $100 \mathrm{~s}^{-1}$ for $\geq 5 \mathrm{~min}$ to ensure that the high viscosity regime was reached and shear-induced phases formed [4-6]. Relaxation of shear-induced structures was minimized by constructing and vitrifying the sandwich within $30 \mathrm{sec}$ of stopping the rheometer, much shorter than the $\sim 100 \mathrm{sec}$ relaxation time [4-6]. Vitrified water was sublimated from the fractured surfaces at $10^{-5} \mathrm{~Pa}$ and $-110^{\circ} \mathrm{C}$ for 30-90 s leaving surfactant structures protruding $6-$ $20 \mathrm{~nm}$ from the background. Replicas were made by depositing $2.5 \mathrm{~nm}$ of platinum at $45^{\circ}$ to the fracture surface and then $30-50 \mathrm{~nm}$ of carbon normal to the surface [17]. Replicas were cleaned [20], then viewed in a JEOL 100CX transmission electron microscope (TEM) at $100 \mathrm{keV}$. Because of different sublimation rates near micelles and to shadowing effects of the $\mathrm{Pt} / \mathrm{C}$ deposition, micelles appear wider in the micrographs than they truly are, $\sim 20 \mathrm{~nm}$ instead of $5 \mathrm{~nm}$.

Figure 1(a) is a freeze-fracture electron micrograph of unsheared $10 \mathrm{mM}$ TTAA/NaSal showing dark strings of wormlike micelles against a lighter background of water. The micelles are entangled and have no preferred orientation. Figure $1(\mathrm{~b})$ is a tracing highlighting the micelles in Fig. 1(a). Broken lines in Fig. 1(b) represent wormlike micelles which most likely meander in and out of the fracture plane. A very long micelle $(>1 \mu \mathrm{m})$ is shown in a black arc at the base of Fig. 1(b). Apparent junctions are probably overlapping, entangled micelles (e.g., Fig. 1, " $X$ " at small arrow). Dots mark where wormlike micelles pierce the plane end-on [Fig. 1(a), inside circle]. A wide range of curvatures of wormlike micelles exists, from a radius of $50 \mathrm{~nm}$ (Fig. 1 at large arrow) to several microns [black arc, bottom of Fig. 1(b)]. Similar wormlike micelles are seen by cryo-TEM [1]. Overall, the micelles in Fig. 1 have a long persistence length suggesting a high bending rigidity. It is unlikely that electrostatic repulsion prevents the wormlike micelles from bending because the Debye length is only $\sim 30 \AA$ in $10 \mathrm{mM}$ monovalent solutions. Moreover, the micelles are not strongly charged. NMR results indicate that the degree of $\mathrm{Sal}^{-}$binding to the micelle is $\sim 92 \%$ at $25^{\circ} \mathrm{C}$ [21].

After shear, we observe three coexisting morphologies: isotropic wormlike micelles similar to those in Fig. 1, aligned wormlike micelles, and new structures. Aligned strings of micelles appear in Fig. 2, oriented from the top left to the bottom right. The arrow marks where micronslong wormlike micelles overlap. From flow birefringence measurements, the micelles are aligned with the shear flow

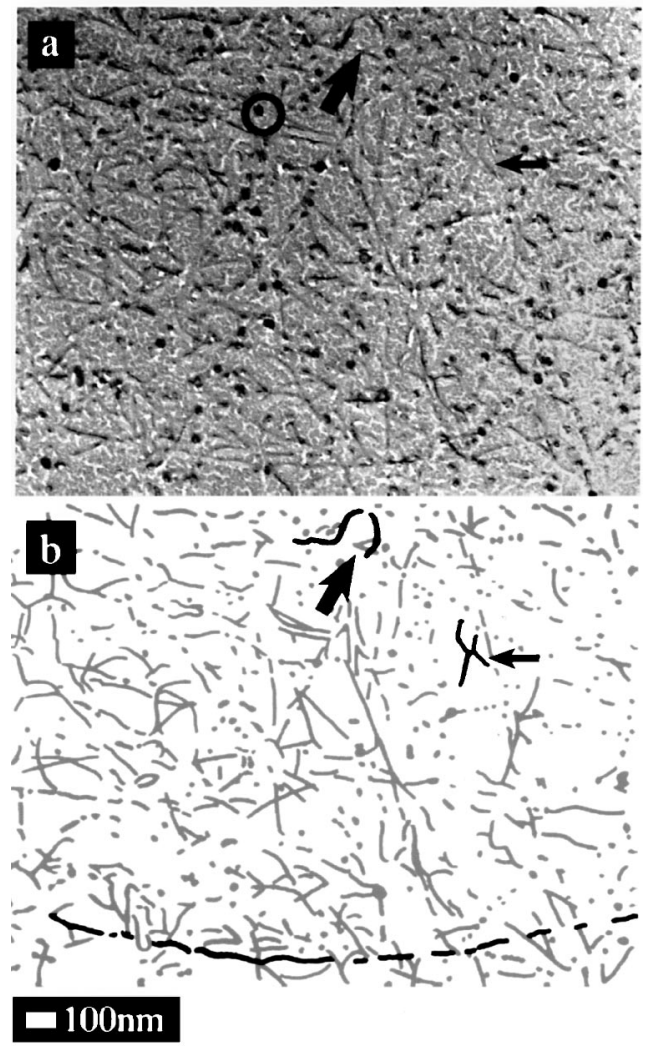

FIG. 1. Freeze-fracture micrograph of unsheared, isotropic wormlike micelles in $10 \mathrm{mM}$ TTAA/NaSal. (b) is a tracing highlighting the micelles in (a). Broken lines (b) represent micelles meandering in and out of the fracture plane. A long micelle $(>1 \mu \mathrm{m})$ is shown at the black arc [bottom of (b)]. Apparent junctions are probably overlapping, entangled micelles [e.g., (a) and (b), "X" at small arrow]. Dots mark where micelles pierce the plane end-on [(a), inside circle]. A wide range of curvatures exists, from a radius of $50 \mathrm{~nm}$ [(a) and (b) at large arrow] to several microns [arc, bottom of (b)].

$[4,5,8,9]$. The density of aligned wormlike micelles does not differ significantly from that of unaligned micelles.

In addition to aligning wormlike micelles, shear also forms new structures shown at high magnification (Fig. 3, $1.37 \mathrm{mM} \mathrm{CTAB} / \mathrm{NaSal}$ ), medium magnification (Fig. 4, $10 \mathrm{mM}$ TTAA/NaSal), and low magnification (Fig. 5, $1.37 \mathrm{mM} \mathrm{CTAB} / \mathrm{NaSal}$ ). Figure 3 shows a peninsula $\sim 0.5 \mu \mathrm{m}$ wide and microns long filled with $30-100 \mathrm{~nm}$ spongelike bumps. The background has a cracked, parched-earth texture (Fig. 3, stars). This texture is observed in FFEM micrographs of water which has been sublimated and indicates little surfactant is present. Areas with high surfactant concentration do not sublimate and appear smooth (Fig. 3, arrow). This suggests that shear concentrates surfactant in the new structure. Semidilute polymer solutions also reportedly segregate under shear into polymer-rich and poor regions [22-24].

The peninsula in Fig. 3 is part of a larger structure, similar to that in Fig. 4. In Fig. 4, an uneven, micronwide strip of new structure runs from the top left to the bottom right. This strip is surrounded by a light grey solution with white strings of wormlike micelles which are 


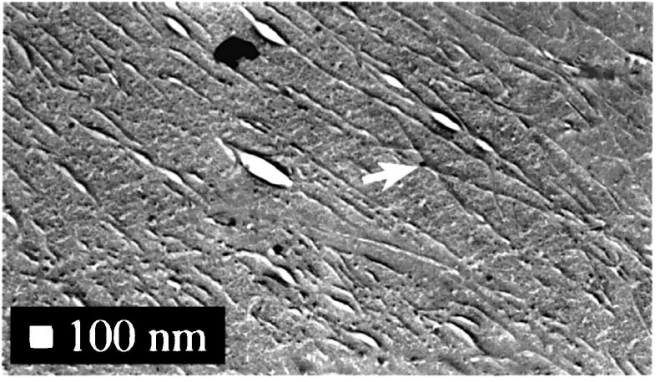

FIG. 2. Sheared $10 \mathrm{mM}$ TTAA/NaSal wormlike micelles (arrow) aligned from the top left to the bottom right.

$\sim 100 \mathrm{~nm}$ long in this cut and rarely overlap (Fig. 4, stars). The new structure sometimes entraps pockets of micellar solution (Fig. 4, triangle) and is uniformly stippled with $10-50 \mathrm{~nm}$ pock marks. Unlike Fig. 3, the background parched-earth texture is not depleted of wormlike micelles. Nevertheless, the texture of the new structure in Fig. 4 is very different from the background micellar solution. The boundary between the new structure and the micellar solution is jagged and stark white borders (arrow), which are rips in the metal replica, guide the eye along it. Ripping and rejoining of the surfactant-rich structure in Fig. 4 would create dramatic temporal fluctuations in the stress, consistent with rheological observations [3-6,9,10]. Hence, we associate the new structures in Figs. 3-5 with the viscous shear-induced structures correlated with shear thickening $[3,10]$.

The strip in Fig. 4 is part of an even larger structure, similar to that in Fig. 5. Stippled, micron-sized patches of the new structure are grouped in a $\sim 4 \mu \mathrm{m}$ swath against a background with a parched-earth texture (Fig. 5, star). Such groups of patches can span hundreds of microns. Typically, the patches of new structure occupy 5\%-10\% of the fracture area. Assuming the patches are uniform in three dimensions, this area corresponds to $\sim 1 \%-2 \%$ of the volume. Although the sample is mostly filled with wormlike micelles, the patches of new structure are easy to locate and identify. The micrographs are two-dimensional slices

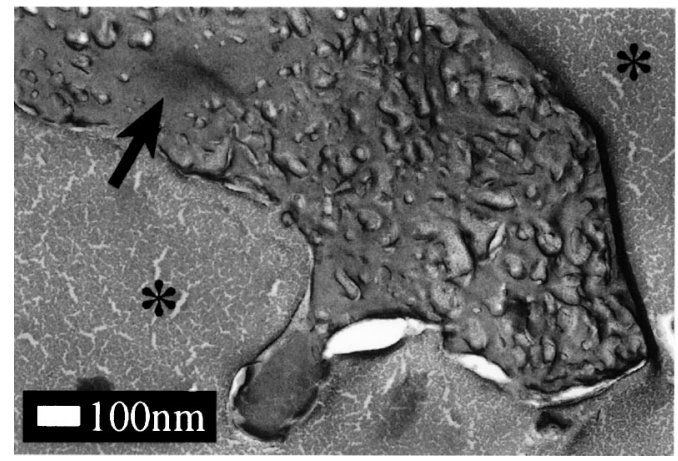

FIG. 3. High magnification new structure in $1.37 \mathrm{mM}$ $\mathrm{CTAB} / \mathrm{NaSal}$. The smooth region (arrow) probably contains more surfactant than the background. The cracked, parchedearth texture of the background (stars) is indicative of water which has been sublimated. No individual micelles appear.

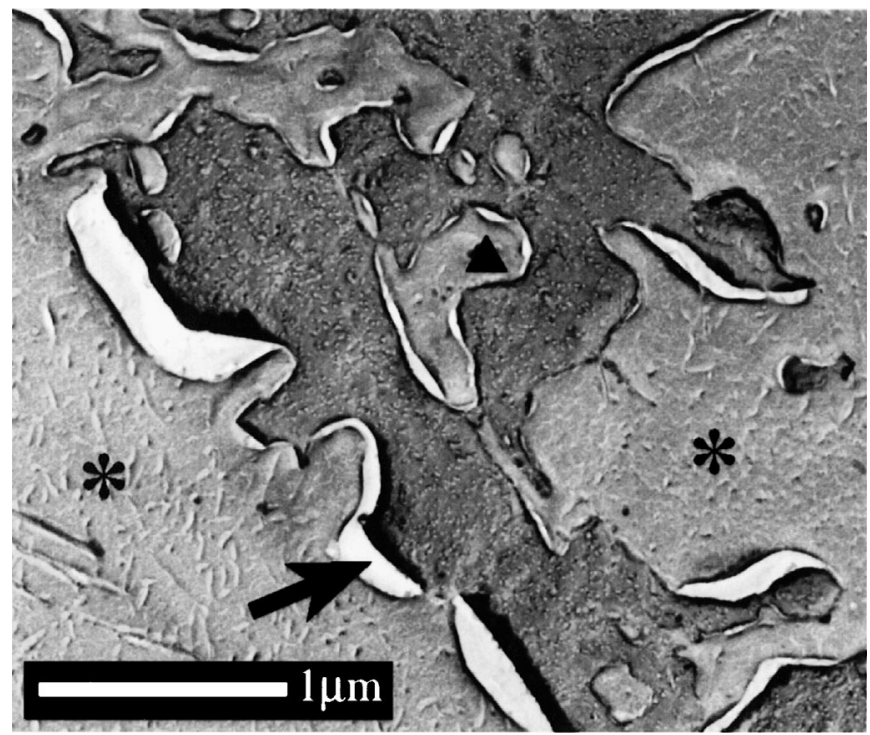

FIG. 4. Pock-marked stripe of new structure in sheared $10 \mathrm{mM}$ TTAA/NaSal against a background wormlike micelles (stars). The arrow marks a rip at the white, jagged border of the new structure and micellar solution. The triangle marks a pocket of micellar solution entrapped in the new structure.

through a three-dimensional sample, and it is reasonable that individual patches are part of a three-dimensional network superstructure. Indeed, this is consistent with light scattering microscopy which shows that shear-induced structures span the sample [10].

Shear-induced structures are birefringent and aligned in the flow direction $[4,5,8,9]$. By contrast, the new structures in Figs. 3-5 have no preferred orientation. This is not surprising since the shear is stopped in order to vitrify the samples and shear-induced structures are known to recoil upon cessation of shear [3]. The recoil would be expected to destroy the alignment of the sample and result in the unoriented structures we observe.

In most cases, the boundary between new structures and micelles is sharp. In contrast, Fig. 6 illustrates a

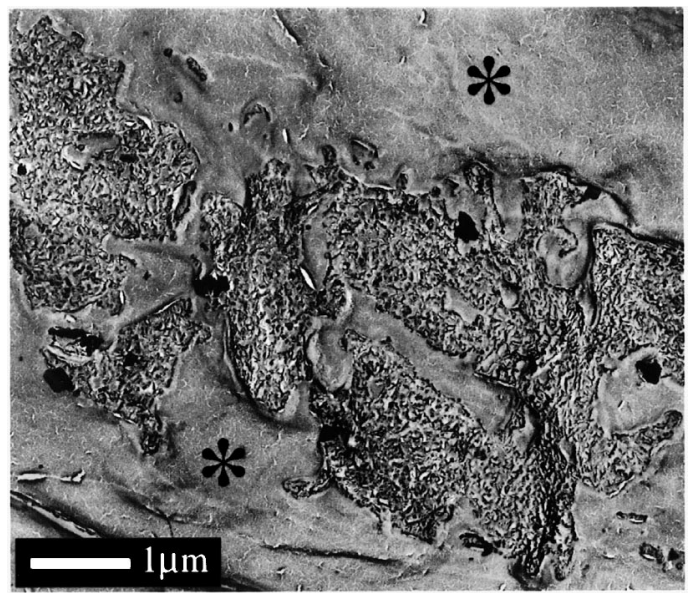

FIG. 5. Low magnification stippled patches of new structure in sheared $1.37 \mathrm{mM} \mathrm{CTAB} / \mathrm{NaSal}$ against a background wormlike micellar solution (stars). 

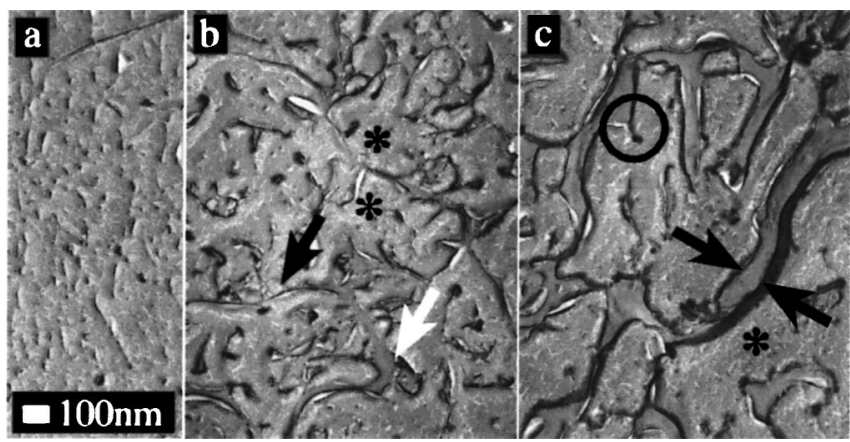

FIG. 6. Transition from isotropic micelles (a) to entangled micelles [(b), black arrow] and to new structure [(b), white arrow, and (c), between arrows] in sheared $10 \mathrm{mM}$ TTAA/NaSal. In (c), the black arrowheads are separated by $\sim 100 \mu \mathrm{m}$. The background of water and individual micelles has a parchedearth texture (stars). The circle marks a cusp.

gradual transition from individual wormlike micelles [Fig. 6(a)] to new structures [Fig. 6(c)] in sheared $10 \mathrm{mM}$ TTAA/NaSal. In the transition region [Fig. 6(b)], the black arrow points to a filigree of entangled individual wormlike micelles. Again, the water background has a parched-earth texture (Fig. 6, stars). The transition region also contains small smooth-textured structures [Fig. 6(b), white arrow]. In Fig. 6(c), these are elongated and often hundreds of nanometers wide (between the black arrowheads). In contrast, an individual micelle is $5 \mathrm{~nm}$ wide.

Recently it has been shown that lamellar phases can be formed by adding excess salt to micellar solutions [25]. Similarly, it is possible that shear segregates micellar solutions into surfactant-rich and poor regions which spontaneously form new structures. To investigate this possibility, we searched for new structures in unsheared solutions at high surfactant concentrations. No new structures were discovered in unsheared $13.7 \mathrm{mM} \mathrm{CTAB} / \mathrm{NaSal}$ at room temperature, implying that condensing the sample by 10 times is not sufficient.

While the FFEM micrographs unambiguously demonstrate that the new structures and micellar solutions have very different morphologies, there remains the question of exactly what kind of structure is formed. Figures 35 show no evidence that the new structure is comprised of micelles, either entangled, branched, or in bundles. The bumpy structures in Figs. 3 and 5 and the smooth-textured elongated structures in Fig. 6(c) resemble sponge phases of bilayers [26]. The pock-marked structures in Fig. 4 are reminiscent of perforated lamellae, although these putative lamellae are never seen edge-on. Whatever the precise microstructure is, the new structures presented here are consistent with previous scattering results [3-10].

Overall, the micrographs of Figs. 3-5 are consistent with models that the shear-induced phase is highly entangled [6] viscous [10], strongly aligned, and consists of structures much larger than individual micelles $[8,15]$. The micrographs do not support proposals that the struc- tures are only stringlike [15], pearl strings of micelles, or pseudonematic domains [8]. The density of aligned, sheared micelles approximates that of unaligned micelles (Figs. 1 and 2), suggesting that aligned micelles alone cannot constitute the highly scattering shear-induced structures correlated with shear thickening $[3,6,10]$.

To summarize, shear forms new micron-sized structures which are much larger than individual wormlike micelles and consistent with light scattering results $[3,6,10]$. Patches of the new structure connect to form network superstructures that span hundreds of microns. These percolating superstructures are likely responsible for the increase in viscosity, consistent with the shear thickening observed [3-6,8-10]. The new structures are richer in surfactant than the surrounding micellar solution and have a submicron stippled or spongelike texture. Two other morphologies coexist with the new structures, aligned and unaligned wormlike micelles.

This work was supported by the NSF 9319447 and the MSERC program of the NSF under DMR-96-32716.

[1] T. M. Clausen et al., J. Phys. Chem. 96, 474 (1992).

[2] M. E. Cates and S. J. Candau, J. Phys. Condens. Matter 2, 6869 (1990).

[3] C.-H. Liu and D. J. Pine, Phys. Rev. Lett. 77, 2121 (1996).

[4] Y. Hu et al., J. Rheol. 37, 531 (1993).

[5] Y. Hu et al., Langmuir 10, 80 (1994).

[6] P. Boltenhagen et al., Europhys. Lett. 38, 389 (1997).

[7] M. Y. Lin et al., Physica (Amsterdam) 213\&214B, 613 (1995).

[8] S. Hofmann et al., Ber. Bunsen-Ges. Phys. Chem. 95, 153 (1991).

[9] I. Wunderlich et al., Rheol. Acta 26, 532 (1987).

[10] P. Boltenhagen et al., Phys. Rev. Lett. 79, 2359 (1997).

[11] Y. T. Hu et al., J. Colloid Interface Sci. 156, 31 (1993).

[12] S.-Q. Wang, Macromolecules 25, 7003 (1992).

[13] R. Bruinsma et al., J. Chem. Phys. 96, 7710 (1992).

[14] M.E. Cates and M.S. Turner, Europhys. Lett. 11, 681 (1990).

[15] E. K. Wheeler et al., Rheol. Acta 35, 139 (1996).

[16] N. A. Spenley et al., Phys. Rev. Lett. 71, 939 (1993).

[17] J. A. Zasadzinski and S. M. Bailey, J. Electron. Microsc. Tech. 13, 309 (1989).

[18] TTAA (ethoquad T/13-50) is from AKZO (MW $454 \mathrm{~g} / \mathrm{mol})$. Its nonlinear rheological behavior has been characterized [Y. T. Hu and E. F. Matthys, Rheol. Acta 34, 450 (1995)]. CTAB is from Sigma (MW $364.5 \mathrm{~g} / \mathrm{mol}$ ).

[19] L. J. Magid et al., Langmuir 6, 1609 (1990).

[20] R. Fetter and M. Costello, J. Microsc. 141, 277 (1986).

[21] U. Olsson et al., J. Phys. Chem. 90, 5223 (1986).

[22] H. Yanase et al., Rheol. Acta 30, 89 (1991).

[23] X.-L. Wu et al., Phys. Rev. Lett. 66, 2408 (1991).

[24] P. K. Dixon et al., Phys. Rev. Lett. 68, 2239 (1992).

[25] J. Candau et al., Langmuir (to be published).

[26] R. Strey et al., Langmuir 6, 1635 (1990). 